Disclosure of Interests: None declared

DOI: 10.1136/annrheumdis-2020-eular.3910

\section{AB0872 1 EFFICACY AND SAFETY OF THE COMBINATION OF APOCYNIN AND PAEONOL (APPA) IN PATIENTS WITH OSTEOARTHRITIS: AN UNCONTROLLED PATIENT CASE SERIES}

\section{N. Larkins ${ }^{1} .{ }^{1} A K L$ Research and Development Ltd, Stevenage, United Kingdom}

Background: Apocynin and paeonol are secondary metabolites of plants used in traditional Asian medicine for centuries. The combination of synthetic versions of these two molecules (APPA) was developed initially for the treatment of osteoarthritis (OA) in animals where it has been found at least as effective as meloxicam. Human clinical trials are currently ongoing.

Objectives: To report the outcomes from a case series of patients treated with APPA.

Methods: Subjects with a diagnosis of OA, who had tried unsuccessfully a number of standard therapies, requested treatment with APPA from the author (NL), often following successful treatment of their animals with the combination or via networking. The usual daily dose was $1240 \mathrm{mg}$ paeonol and $352 \mathrm{mg}$ of apocynin taken as two 400mg capsules twice daily.

Results: Twenty-three subjects with a diagnosis of OA of whom 7 were scheduled for surgery have been treated with APPA. There were 10 female and 13 males with an age range from 40 to 81 years. Nine patients had OA of the knees, 5 of whom had bilateral involvement, 7 had hip OA, 5 bilaterally and 2 with end stage bone on bone disease. Four patients had hand OA, one of whom also had disease of the hip, lower back and feet. In 3 patients the joints involved was not recorded. In 19 patients treatment was reported as effective. In 4 patients the treatment was ineffective, all of who were scheduled or had been recommended for surgery. In 2 of these cases cases this was bone on bone The duration of treatment for OA at the last recorded follow-up was reported for 16 patients in whom treatment was deemed effective and ranged from 9 to 120 months (median 24 months). In a further 3 patients the treatment the duration was not reported. In the 4 patients where no benefit was reported APPA was discontinued within a few weeks. In no case was there a report of APPA being discontinued due to adverse events. In 13 patients it was specifically stated that there had been no adverse events whereas for the remaining 10 patients it was not documented whether adverse events had occurred or not.

Conclusion: Treatment with APPA was reported as effective by $82.6 \%$ of patients. In all patients in which the combination was ineffective the disease was severe with joint replacement recommended or scheduled; in two patients this was bone on bone. This would suggest that APPA is not a simple analgesic, a conclusion supported by effects seen in the rat meniscal tear model where possible disease modifying effects have been reported (1).

References:

[1] Glasson, S., Bendele, A. and Larkins, N. (2012). APPA provides disease modification in preclinical osteoarthritis. Osteoarthritis and Cartilage 20: S72 -S73 Abstract 132

Disclosure of Interests: Nicholas Larkins Shareholder of: AKL R and D Ltd,

Employee of: AKL R and D Ltd

DOI: 10.1136/annrheumdis-2020-eular.1221

\section{AB0873 ULTRASOUND GUIDED INTRA-ARTICULAR INJECTION WITH HYALURONIC ACID AGENTS IN MODERATE HIP OSTEOARTHRITIS}

M. Micu ${ }^{1}$, A. Micu², S. Bolboacă ${ }^{3} .{ }^{1}$ Rehabilitation Clinical Hospital Cluj- Napoca, Rheumatology Division, Cluj- Npaoca, Romania; " "luliu Hațieganu"University of Medicine and Pharmacy Cluj-Napoca, Cluj- Napoca, Romania; "'luliu Hațieganu" University of Medicine and Pharmacy Cluj-Napoca, Department of Medical Informatics and Biostatistics, Cluj- Napoca, Romania

Background: Current guidelines usually only include hyaluronic acid (HA) intraarticular injection as an alternative therapy option for knee osteoarthritis (OA) When compared to the blind approach, ultrasound guided intra-articular injections (USGIA) have shown a higher efficacy and reduced number of adverse events due to injection techniques [1]. This has allowed targeting deep joints like the hip. The objective of the study is to evaluate the short and long term efficacy and safety of HA-USGIA in moderate hip OA.

Methods: Patients with Kellgren-Lawrence II and III hip OA (refractory to NSAIDs, pain killers and chondroprotective agents) were prospectively recruited to receive 3 consecutive weekly doses of HA- USGIA using free hand technique [2]. Informed written consent was signed. VAS pain scale and WOMAC score was performed at baseline and at 3 and 6 months after the end of treatment. The first injection was performed after a 1-month wash out. $\mathrm{X}$ ray assessment was made at baseline and after 6 months. Ultrasound evaluation was made at baseline and at each visit.
Results: Fifteen patients (median age 67 years, IQR 63-69 years, 13/15 women) with hip OA were enrolled and 28 hip joints were injected (Fig 1a,b) None of the hips presented ultrasound detected capsular distension (suggesting inflammation) during the study. Table 1 presents the results on medication efficacy at 3 and 6 months. The pain evaluated by VAS score showed a significant and progressive decrease from baseline to 3 and 6 months respectively (Table 1 ). Indeed, a significant and sustained decrease of total WOMAC scores and its separate domains- pain, stiffness and function was recorded from baseline to 3 and 6 months (Fig 2a,b). No significant changes were observed regarding the WOMAC score stiffness and function domains when comparing results at 3 and $6 \mathrm{~ms}$. Moreover, WOMAC-pain score was significantly lower at $6 \mathrm{~ms}$ as compared to $3 \mathrm{~ms}$ (Table 1), highlighting the dramatic and sustained medication impact on the most relevant paramete for clinical practice, in patients with hip OA. Neither drug-related nor injection technique related adverse effects were recorded. No patient developed signs of hip osteonecrosis or inflammatory lesions during follow-up.

Table 1. The trends of the scores expressed as medians and comparisons between follow-ups

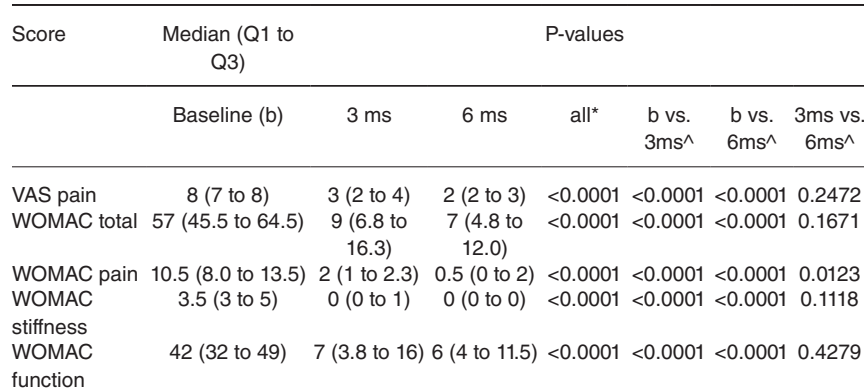

function

Q1 = first quartile; Q3 = third quartile

* Friedman test; ^ Wilcoxon test; 3 ms-3 months, 6 ms-6 months, b-baseline

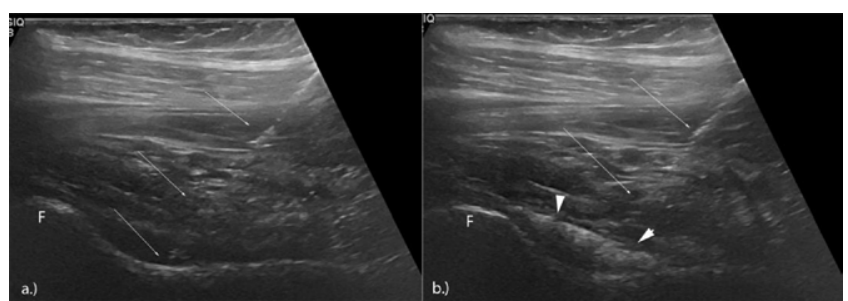

Fig.1. Legend: a.) Longitudinal scanning at hip joint level. F- femoral head, arrows showing the hyperechoic needle penetrating the muscles and arriving inside the anterior join recess. b.) Post-procedural longitudinal scanning at the hip joint level. Arrowheads- showing the hyperechoic intra-articular drug moving anti-gravitational and distending the capsule.

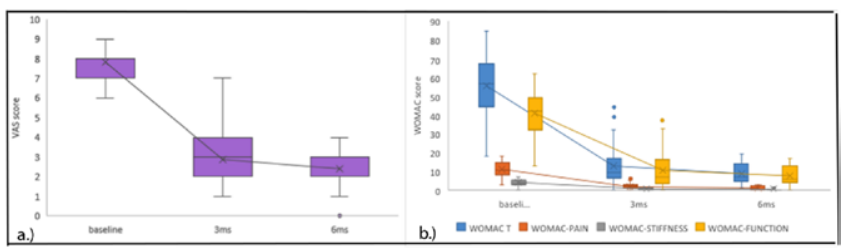

Fig 2. ab Legend. a.) Evolution of VAS for pain over time; b.) Evolution of WOMAC total score and its components over time. The line in the box is the value of median, the box is the first and the third quartile, the wishers are the minimum and maximum and the $x$ is the value of mean. $3 \mathrm{~ms}-3$ months, $6 \mathrm{~ms}-6$ months, WOMAC T- WOMAC total.

Conclusion: The results suggest that HA - USGIA may be an effective and safe treatment for moderate hip OA, due to its short and long term benefits. This treatment should not be delayed until advanced $O A$ is diagnosed. Longitudinal controlled studies on larger cohorts are warranted to confirm these preliminary results.

\section{References:}

[1] Migliore A, Giovannangeli F, Granata M and Lagana B. Hyalgan G-F 20: Review of its Safety and Efficacy in the Management of Joint Pain in Osteoarthritis. Clinical Medical Insights: Arthritis and Musculoskeletal Disorders 2010;3: 55-68. 